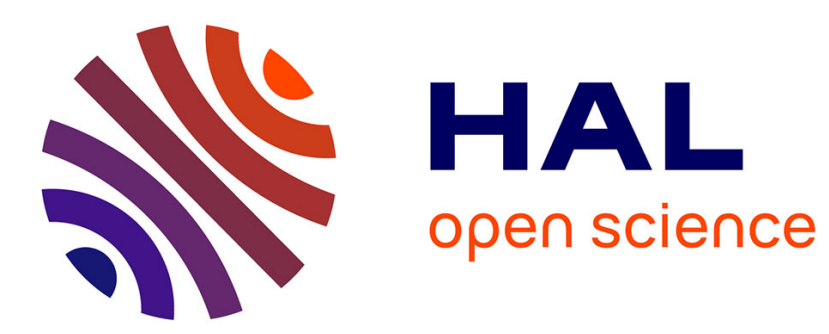

\title{
Existence of stationary solutions for some nonlocal reaction-diffusion equations
}

Vitali Vougalter, Vitaly Volpert

\section{To cite this version:}

Vitali Vougalter, Vitaly Volpert. Existence of stationary solutions for some nonlocal reaction-diffusion equations. Dynamics of Partial Differential Equations, 2015, 10.4310/DPDE.2015.v12.n1.a3 . hal01237921

\section{HAL Id: hal-01237921 \\ https://hal.science/hal-01237921}

Submitted on 8 Dec 2015

HAL is a multi-disciplinary open access archive for the deposit and dissemination of scientific research documents, whether they are published or not. The documents may come from teaching and research institutions in France or abroad, or from public or private research centers.
L'archive ouverte pluridisciplinaire HAL, est destinée au dépôt et à la diffusion de documents scientifiques de niveau recherche, publiés ou non, émanant des établissements d'enseignement et de recherche français ou étrangers, des laboratoires publics ou privés. 


\title{
EXISTENCE OF STATIONARY SOLUTIONS FOR SOME NONLOCAL REACTION-DIFFUSION EQUATIONS
}

\author{
Vitali Vougalter $^{1}$, Vitaly Volpert ${ }^{2}$ \\ ${ }^{1}$ Department of Mathematics, University of Toronto \\ Toronto, Ontario, M5S 2E4, Canada \\ e-mail: vitali@math.toronto.edu \\ ${ }^{2}$ Institute Camille Jordan, UMR 5208 CNRS, University Lyon 1 \\ Villeurbanne, 69622, France \\ e-mail: volpert@math.univ-lyon1.fr
}

\begin{abstract}
The paper is devoted to the existence of solutions of a nonlocal reactiondiffusion equation arising in population dynamics. The proof is based on a fixed point technique. We use solvability conditions for elliptic operators in unbounded domains which do not satisfy the Fredholm property.
\end{abstract}

AMS Subject Classification: 35J05, 35P30, 47F05

Key words: nonlinear heat equations, non Fredholm operators, Sobolev spaces

\section{Introduction}

In this work we study the existence of stationary solutions of the integro-differential equation

$$
\frac{\partial u}{\partial t}=D \Delta u+\int_{\mathbb{R}^{d}} K(x-y) g(u(y, t)) d y+f(x)
$$

arising in cell population dynamics. The space variable $x$ corresponds to the cell genotype, $u(x, t)$ is the cell density as a function of their genotype and time. The right-hand side of this equation describes the evolution of cell density due to cell proliferation, mutations and cell influx. More precisely, the diffusion term corresponds to the change of genotype due to small random mutations, while the integral term describes large mutations. Here $g(u)$ is the rate of cell birth which depends on $u$ (density dependent proliferation), and the function $K(x-y)$ shows the proportion of newly born cells which change their genotype from $y$ to $x$. We suppose that it depends on the distance between the genotypes. Finally, the last term in the right-hand side of this equation describes the influx of cells for different genotypes. 
In what follows we will set $D=1$ and will study the existence of solutions of the equation

$$
\Delta u+\int_{\mathbb{R}^{d}} K(x-y) g(u(y)) d y+f(x)=0 .
$$

We will consider the case where the linear part of this operator does not satisfy the Fredholm property and conventional methods of nonlinear analysis may not be applicable. We will use solvability conditions for non Fredholm operators and will use the method of contracting mappings.

Consider the equation

$$
-\Delta u+V(x) u-a u=f,
$$

where $u \in E=H^{2}\left(\mathbb{R}^{d}\right)$ and $f \in F=L^{2}\left(\mathbb{R}^{d}\right), d \in \mathbb{N}, a$ is a constant and the scalar potential function $V(x)$ either vanishes or tends to 0 at infinity. When $a \geq 0$, the essential spectrum of the operator $A: E \rightarrow F$ corresponding to the left side of problem (1.3) contains the origin. As a consequence, such operator does not satisfy the Fredholm property. Its image is not closed, for $d>1$ the dimension of its kernel and the codimension of its image are not finite. The present article is devoted to the studies of some properties of the operators of this kind. Note that elliptic equations containing non Fredholm operators were studied actively in recent years. Approaches in weighted Sobolev and Hölder spaces were developed in [2], [3], [4], [5], [6]. The non Fredholm Schrödinger type operators were treated using the methods of the spectral and the scattering theory in [12], [14], [15], [16], [18]. The Laplacian operator with drift from the perspective of the operators without Fredholm property was studied in [17] and linearized Cahn-Hilliard problems in [19] and [21]. Nonlinear non Fredholm elliptic equations were treated in [20] and [22]. Important applications to the theory of reaction-diffusion problems were explored in [9], [10]. Operators without Fredholm property arise also when treating wave systems with an infinite number of localized traveling waves (see [1]). Particularly, when $a=0$ the operator $A$ is Fredholm in some properly chosen weighted spaces (see [2], [3], [4], [5], [6]). However, the case of $a \neq 0$ is significantly different and the approach developed in these articles cannot be used.

We set $K(x)=\varepsilon \mathcal{K}(x)$, where $\varepsilon \geq 0$. We suppose that the following assumption is satisfied.

Assumption 1. Let $f(x): \mathbb{R}^{5} \rightarrow \mathbb{R}$ be nontrivial, $f(x) \in L^{1}\left(\mathbb{R}^{5}\right)$ and $\nabla f(x) \in$ $L^{2}\left(\mathbb{R}^{5}\right)$. Assume also that $\mathcal{K}(x): \mathbb{R}^{5} \rightarrow \mathbb{R}$ and $\mathcal{K}(x) \in L^{1}\left(\mathbb{R}^{5}\right)$.

The choice of the space dimension is related to the solvability conditions for linear elliptic problems in unbounded domains [22]. There are some solvability conditions for $d<5$, and solvability conditions are not required for $d \geq 5$ (see Appendix). We will consider here only the case $d=5$. We will not study the 
problem in dimensions $d>5$ to avoid extra technicalities since the proof will use similar ideas and no orthogonality conditions for the solvability of equation (1.8) are required analogously to $d=5$ (Lemma 7 of [22]). From the point of view of applications, the space dimension is not limited to $d=3$ since the space variable corresponds to cell genotype and not the usual physical space.

By virtue of the Sobolev inequality (see e.g. p.183 of [11]) under the assumption above we have

$$
f(x) \in L^{2}\left(\mathbb{R}^{5}\right) .
$$

Let us consider the Sobolev space

$$
H^{3}\left(\mathbb{R}^{5}\right)=\left\{u(x): \mathbb{R}^{5} \rightarrow \mathbb{C} \mid u(x) \in L^{2}\left(\mathbb{R}^{5}\right),(-\Delta)^{\frac{3}{2}} u \in L^{2}\left(\mathbb{R}^{5}\right)\right\}
$$

with the norm

$$
\|u\|_{H^{3}\left(\mathbb{R}^{5}\right)}^{2}:=\|u\|_{L^{2}\left(\mathbb{R}^{5}\right)}^{2}+\left\|(-\Delta)^{\frac{3}{2}} u\right\|_{L^{2}\left(\mathbb{R}^{5}\right)}^{2} .
$$

The operator $(-\Delta)^{\frac{3}{2}}$ is defined by means of the spectral calculus. By virtue of the Sobolev embedding we have

$$
\|u\|_{L^{\infty}\left(\mathbb{R}^{5}\right)} \leq c_{e}\|u\|_{H^{3}\left(\mathbb{R}^{5}\right)}
$$

Here $c_{e}>0$ is the constant of the embedding. The hat symbol will denote the standard Fourier transform, namely

$$
\widehat{u}(p)=\frac{1}{(2 \pi)^{\frac{5}{2}}} \int_{\mathbb{R}^{5}} u(x) e^{-i p x} d x .
$$

Let us express the Sobolev norm as

$$
\|u\|_{H^{3}\left(\mathbb{R}^{5}\right)}^{2}=\int_{\mathbb{R}^{5}}\left(1+|p|^{6}\right)|\widehat{u}(p)|^{2} d p .
$$

When the nonnegative parameter $\varepsilon=0$, we obtain at the standard Poisson equation

$$
-\Delta u=f(x) .
$$

Assumption 1 via Lemma 7 of [22] implies that problem (1.8) has a unique solution $u_{0}(x) \in H^{2}\left(\mathbb{R}^{5}\right)$ such that no orthogonality conditions are required, and

$$
\nabla(-\Delta u)=\nabla f(x) \in L^{2}\left(\mathbb{R}^{5}\right) .
$$

Hence, for the unique solution of our linear equation (1.8) we obtain $u_{0}(x) \in$ $H^{3}\left(\mathbb{R}^{5}\right)$. Let us look for the resulting solution of the nonlinear problem (1.2) as

$$
u(x)=u_{0}(x)+u_{p}(x) .
$$


Obviously, we arrive at the perturbative equation

$$
-\Delta u_{p}=\varepsilon \int_{\mathbb{R}^{5}} \mathcal{K}(x-y) g\left(u_{0}(y)+u_{p}(y)\right) d y .
$$

We introduce a closed ball in the Sobolev space

$$
B_{\rho}:=\left\{u(x) \in H^{3}\left(\mathbb{R}^{5}\right) \mid\|u\|_{H^{3}\left(\mathbb{R}^{5}\right)} \leq \rho\right\}, \quad 0<\rho \leq 1 .
$$

Let us look for the solution of (1.10) as the fixed point of the auxiliary nonlinear equation

$$
-\Delta u=\varepsilon \int_{\mathbb{R}^{5}} \mathcal{K}(x-y) g\left(u_{0}(y)+v(y)\right) d y
$$

in ball (1.11). For a given function $v(y)$ it is an equation with respect to $u(x)$. The left side of (1.12) contains the non Fredholm operator $-\Delta: H^{2}\left(\mathbb{R}^{5}\right) \rightarrow L^{2}\left(\mathbb{R}^{5}\right)$, since its essential spectrum fills the nonnegative semi-axis $[0,+\infty)$ and therefore, this operator has no bounded inverse. The analogous situation appeared in [20] and [22] but as distinct from the present work, the problems studied there required orthogonality relations. The fixed point technique was exploited in [13] to estimate the perturbation to the standing solitary wave of the Nonlinear Schrödinger (NLS) equation when either the external potential or the nonlinear term in the NLS were perturbed but the Schrödinger type operator involved in the nonlinear equation had the Fredholm property (see Assumption 1 of [13], also [7]). We define the interval on the real line

$$
I:=\left[-c_{e}\left\|u_{0}\right\|_{H^{3}\left(\mathbb{R}^{5}\right)}-c_{e}, c_{e}\left\|u_{0}\right\|_{H^{3}\left(\mathbb{R}^{5}\right)}+c_{e}\right] .
$$

Let us make the following assumption about the nonlinear part of equation (1.2).

Assumption 2. Let $g(s): \mathbb{R} \rightarrow \mathbb{R}$, such that $g(0)=0$ and $g^{\prime}(0)=0$. We also assume that $g(s) \in C_{2}(\mathbb{R})$, such that

$$
a_{2}:=\sup _{s \in I}\left|g^{\prime \prime}(s)\right|>0 .
$$

Clearly $a_{1}:=\sup _{s \in I}\left|g^{\prime}(s)\right|>0$ as well, otherwise the function $g(s)$ will be constant on the interval $I$ and $a_{2}=0$. For example, $g(s)=s^{2}$ evidently satisfies the assumption above.

We introduce the operator $T_{g}$ such that $u=T_{g} v$, where $u$ is a solution of equation (1.12). Our main statement is as follows.

Theorem 3. Let Assumptions 1 and 2 hold. Then problem (1.12) defines the map $T_{g}: B_{\rho} \rightarrow B_{\rho}$, which is a strict contraction for all $0<\varepsilon<\varepsilon *$ for a certain $\varepsilon *>0$. The unique fixed point $u_{p}(x)$ of the map $T_{g}$ is the only solution of equation (1.10) in $B_{\rho}$. 
Obviously the resulting solution of problem (1.2) given by (1.9) will be nontrivial since the source term $f(x)$ is nontrivial and $g(0)=0$ due to our assumptions. Let us make use of the following trivial technical lemma.

Lemma 4. Consider the function $\varphi(R):=\alpha R+\frac{\beta}{R^{4}}$ for $R \in(0,+\infty)$, where the constants $\alpha, \beta>0$. It attains the minimal value at $R^{*}=\left(\frac{4 \beta}{\alpha}\right)^{\frac{1}{5}}$, which is given by $\varphi\left(R^{*}\right)=\frac{5}{4^{\frac{4}{5}}} \alpha^{\frac{4}{5}} \beta^{\frac{1}{5}}$.

We proceed to the proof of our main statement.

\section{The existence of the perturbed solution}

Proof of Theorem 3. We choose an arbitrary $v(x) \in B_{\rho}$ and denote the term involved in the integral expression in right side of problem (1.12) as $G(x):=g\left(u_{0}+v\right)$. Let us apply the standard Fourier transform (1.6) to both sides of equation (1.12). We obtain

$$
\widehat{u}(p)=\varepsilon(2 \pi)^{\frac{5}{2}} \frac{\widehat{\mathcal{K}}(p) \widehat{G}(p)}{p^{2}} .
$$

Thus for the norm we have

$$
\|u\|_{L^{2}\left(\mathbb{R}^{5}\right)}^{2}=(2 \pi)^{5} \varepsilon^{2} \int_{\mathbb{R}^{5}} \frac{|\widehat{\mathcal{K}}(p)|^{2}|\widehat{G}(p)|^{2}}{|p|^{4}} d p .
$$

Evidently, for any $G(x) \in L^{1}\left(\mathbb{R}^{5}\right)$

$$
\|\widehat{G}(p)\|_{L^{\infty}\left(\mathbb{R}^{5}\right)} \leq \frac{1}{(2 \pi)^{\frac{5}{2}}}\|G(x)\|_{L^{1}\left(\mathbb{R}^{5}\right)} .
$$

Note that as distinct from works [20] and [22] in lower dimensions, here we do not try to control the norm

$$
\left\|\frac{\widehat{\mathcal{K}}(p)}{p^{2}}\right\|_{L^{\infty}\left(\mathbb{R}^{5}\right)} .
$$

We estimate the right side of (2.1) using (2.2) with $R>0$ as

$$
\begin{aligned}
& (2 \pi)^{5} \varepsilon^{2} \int_{|p| \leq R} \frac{|\widehat{\mathcal{K}}(p)|^{2}|\widehat{G}(p)|^{2}}{|p|^{4}} d p+(2 \pi)^{5} \varepsilon^{2} \int_{|p|>R} \frac{|\widehat{\mathcal{K}}(p)|^{2}|\widehat{G}(p)|^{2}}{|p|^{4}} d p \leq \\
\leq & \varepsilon^{2} \frac{1}{(2 \pi)^{5}}\|\mathcal{K}\|_{L^{1}\left(\mathbb{R}^{5}\right)}^{2}\|G(x)\|_{L^{1}\left(\mathbb{R}^{5}\right)}^{2}\left|S_{5}\right| R+\varepsilon^{2}\|\mathcal{K}\|_{L^{1}\left(\mathbb{R}^{5}\right)}^{2} \frac{1}{R^{4}}\|G(x)\|_{L^{2}\left(\mathbb{R}^{5}\right)}^{2} .
\end{aligned}
$$


Here and further down $S_{5}$ denotes the unit sphere in the space of five dimensions centered at the origin and $\left|S_{5}\right|$ its Lebesgue measure (see e.g. p.6 of [11]). The fact that $v(x) \in B_{\rho}$ yields

$$
\left\|u_{0}+v\right\|_{L^{2}\left(\mathbb{R}^{5}\right)} \leq\left\|u_{0}\right\|_{H^{3}\left(\mathbb{R}^{5}\right)}+1
$$

and the Sobolev embedding (1.5) implies

$$
\left|u_{0}+v\right| \leq c_{e}\left\|u_{0}\right\|_{H^{3}\left(\mathbb{R}^{5}\right)}+c_{e} .
$$

Using the formula $G(x)=\int_{0}^{u_{0}+v} g^{\prime}(s) d s$, with the interval $I$ defined in (1.13), we easily arrive at

$$
|G(x)| \leq \sup _{s \in I}\left|g^{\prime}(s)\right|\left|u_{0}+v\right|=a_{1}\left|u_{0}+v\right|
$$

Therefore,

$$
\|G(x)\|_{L^{2}\left(\mathbb{R}^{5}\right)} \leq a_{1}\left\|u_{0}+v\right\|_{L^{2}\left(\mathbb{R}^{5}\right)} \leq a_{1}\left(\left\|u_{0}\right\|_{H^{3}\left(\mathbb{R}^{5}\right)}+1\right) .
$$

Evidently, $G(x)=\int_{0}^{u_{0}+v} d s\left[\int_{0}^{s} g^{\prime \prime}(t) d t\right]$. Thus, we obtain

$$
\begin{gathered}
|G(x)| \leq \frac{1}{2} \sup _{t \in I}\left|g^{\prime \prime}(t) \| u_{0}+v\right|^{2}=\frac{a_{2}}{2}\left|u_{0}+v\right|^{2}, \\
\|G(x)\|_{L^{1}\left(\mathbb{R}^{5}\right)} \leq \frac{a_{2}}{2}\left\|u_{0}+v\right\|_{L^{2}\left(\mathbb{R}^{5}\right)}^{2} \leq \frac{a_{2}}{2}\left(\left\|u_{0}\right\|_{H^{3}\left(\mathbb{R}^{5}\right)}+1\right)^{2} .
\end{gathered}
$$

Hence we derive the estimate from above for the right side of (2.3) as

$$
\frac{\varepsilon^{2}}{(2 \pi)^{5}}\|\mathcal{K}\|_{L^{1}\left(\mathbb{R}^{5}\right)}^{2}\left|S_{5}\right| \frac{a_{2}^{2}}{4}\left(\left\|u_{0}\right\|_{H^{3}\left(\mathbb{R}^{5}\right)}+1\right)^{4} R+\varepsilon^{2}\|\mathcal{K}\|_{L^{1}\left(\mathbb{R}^{5}\right)}^{2} a_{1}^{2}\left(\left\|u_{0}\right\|_{H^{3}\left(\mathbb{R}^{5}\right)}+1\right)^{2} \frac{1}{R^{4}},
$$

where $R \in(0,+\infty)$. Lemma 4 gives us the minimal value of the expression above. Thus

$$
\|u\|_{L^{2}\left(\mathbb{R}^{5}\right)}^{2} \leq \varepsilon^{2}\|\mathcal{K}\|_{L^{1}\left(\mathbb{R}^{5}\right)}^{2} \frac{\left|S_{5}\right|^{\frac{4}{5}}}{(2 \pi)^{4}} a_{2}^{\frac{8}{5}}\left(\left\|u_{0}\right\|_{H^{3}\left(\mathbb{R}^{5}\right)}+1\right)^{3 \frac{3}{5}} a_{1}^{\frac{2}{5}} \frac{5}{4^{\frac{8}{5}}} .
$$

Obviously, (1.12) yields

$$
\nabla(-\Delta u)=\varepsilon \nabla \int_{\mathbb{R}^{5}} \mathcal{K}(x-y) G(y) d y
$$

and

$$
\nabla G(x)=g^{\prime}\left(u_{0}+v\right)\left(\nabla u_{0}+\nabla v\right) .
$$

Let us make use of the formula

$$
g^{\prime}\left(u_{0}+v\right)=\int_{0}^{u_{0}+v} g^{\prime \prime}(s) d s .
$$


By virtue of the Sobolev embedding (1.5) we have

$$
\left|g^{\prime}\left(u_{0}+v\right)\right| \leq \sup _{s \in I}\left|g^{\prime \prime}(s) \| u_{0}+v\right| \leq a_{2} c_{e}\left(\left\|u_{0}\right\|_{H^{3}\left(\mathbb{R}^{5}\right)}+1\right) .
$$

Let us make use of the inequality, which can be easily obtained via the standard Fourier transform, namely

$$
\|\nabla u\|_{L^{2}\left(\mathbb{R}^{5}\right)} \leq\|u\|_{H^{3}\left(\mathbb{R}^{5}\right)}
$$

We derive

$$
\left\|(-\Delta)^{\frac{3}{2}} u\right\|_{L^{2}\left(\mathbb{R}^{5}\right)}^{2} \leq \varepsilon^{2}\|\mathcal{K}\|_{L^{1}\left(\mathbb{R}^{5}\right)}^{2} a_{2}^{2} c_{e}^{2}\left(\left\|u_{0}\right\|_{H^{3}\left(\mathbb{R}^{5}\right)}+1\right)^{4} .
$$

The definition of the norm (1.4) along with upper bounds (2.4) and (2.6) yield

$$
\|u\|_{H^{3}\left(\mathbb{R}^{5}\right)} \leq \varepsilon\|\mathcal{K}\|_{L^{1}\left(\mathbb{R}^{5}\right)}\left(\left\|u_{0}\right\|_{H^{3}\left(\mathbb{R}^{5}\right)}+1\right)^{2} a_{2}^{\frac{4}{5}} \sqrt{\frac{\left|S_{5}\right|^{\frac{4}{5}}}{(2 \pi)^{4}} a_{1}^{\frac{2}{5}} \frac{5}{4^{\frac{8}{5}}}+a_{2}^{\frac{2}{5}} c_{e}^{2}} \leq \rho
$$

for all positive values of $\varepsilon$ sufficiently small, such that $u(x) \in B_{\rho}$ as well. If for some $v(x) \in B_{\rho}$ there are two solutions $u_{1,2}(x) \in B_{\rho}$ of equation (1.12), their difference $u(x):=u_{1}(x)-u_{2}(x) \in L^{2}\left(\mathbb{R}^{5}\right)$ solves the Laplace's equation. Due to the fact that there are no nontrivial square integrable harmonic functions, $u(x)=0$ a.e. in $\mathbb{R}^{5}$. Thus, problem (1.12) defines a map $T_{g}: B_{\rho} \rightarrow B_{\rho}$ for $\varepsilon>0$ small enough.

Our goal is to show that this map is a strict contraction. Let us choose arbitrary $v_{1,2}(x) \in B_{\rho}$. By means of the argument above $u_{1,2}=T_{g} v_{1,2} \in B_{\rho}$ as well. (1.12) yields

$$
\begin{aligned}
& -\Delta u_{1}=\varepsilon \int_{\mathbb{R}^{5}} \mathcal{K}(x-y) g\left(u_{0}(y)+v_{1}(y)\right) d y, \\
& -\Delta u_{2}=\varepsilon \int_{\mathbb{R}^{5}} \mathcal{K}(x-y) g\left(u_{0}(y)+v_{2}(y)\right) d y .
\end{aligned}
$$

We introduce

$$
G_{1}(x):=g\left(u_{0}+v_{1}\right), \quad G_{2}(x):=g\left(u_{0}+v_{2}\right) .
$$

By applying the standard Fourier transform (1.6) to both sides of equations (2.7) and (2.8), we arrive at

$$
\widehat{u_{1}}(p)=\varepsilon(2 \pi)^{\frac{5}{2}} \frac{\widehat{\mathcal{K}}(p) \widehat{G_{1}}(p)}{p^{2}}, \quad \widehat{u_{2}}(p)=\varepsilon(2 \pi)^{\frac{5}{2}} \frac{\widehat{\mathcal{K}}(p) \widehat{G_{2}}(p)}{p^{2}} .
$$

Let us express the norm

$$
\left\|u_{1}-u_{2}\right\|_{L^{2}\left(\mathbb{R}^{5}\right)}^{2}=\varepsilon^{2}(2 \pi)^{5} \int_{\mathbb{R}^{5}} \frac{|\widehat{\mathcal{K}}(p)|^{2}\left|\widehat{G_{1}}(p)-\widehat{G_{2}}(p)\right|^{2}}{|p|^{4}} d p .
$$


Clearly, it can be bounded from above via (2.2) by

$$
\frac{\varepsilon^{2}\|\mathcal{K}\|_{L^{1}\left(\mathbb{R}^{5}\right)}^{2}}{(2 \pi)^{5}}\left\|G_{1}(x)-G_{2}(x)\right\|_{L^{1}\left(\mathbb{R}^{5}\right)}^{2}\left|S_{5}\right| R+\frac{\varepsilon^{2}\|\mathcal{K}\|_{L^{1}\left(\mathbb{R}^{5}\right)}^{2}}{R^{4}}\left\|G_{1}(x)-G_{2}(x)\right\|_{L^{2}\left(\mathbb{R}^{5}\right)}^{2},
$$

where $R \in(0,+\infty)$. We will make use of the identity

$$
G_{1}(x)-G_{2}(x)=\int_{u_{0}+v_{2}}^{u_{0}+v_{1}} g^{\prime}(s) d s .
$$

Hence

$$
\left|G_{1}(x)-G_{2}(x)\right| \leq \sup _{s \in I}\left|g^{\prime}(s)\right|\left|v_{1}-v_{2}\right|=a_{1}\left|v_{1}-v_{2}\right|,
$$

such that

$$
\left\|G_{1}(x)-G_{2}(x)\right\|_{L^{2}\left(\mathbb{R}^{5}\right)} \leq a_{1}\left\|v_{1}-v_{2}\right\|_{L^{2}\left(\mathbb{R}^{5}\right)} \leq a_{1}\left\|v_{1}-v_{2}\right\|_{H^{3}\left(\mathbb{R}^{5}\right)} .
$$

Obviously,

$$
G_{1}(x)-G_{2}(x)=\int_{u_{0}+v_{2}}^{u_{0}+v_{1}} d s\left[\int_{0}^{s} g^{\prime \prime}(t) d t\right] .
$$

We estimate $G_{1}(x)-G_{2}(x)$ in the absolute value from above by

$$
\frac{1}{2} \sup _{t \in I}\left|g^{\prime \prime}(t)\right|\left|\left(v_{1}-v_{2}\right)\left(2 u_{0}+v_{1}+v_{2}\right)\right|=\frac{a_{2}}{2}\left|\left(v_{1}-v_{2}\right)\left(2 u_{0}+v_{1}+v_{2}\right)\right| .
$$

The Schwarz inequality yields the upper bound for the norm $\left\|G_{1}(x)-G_{2}(x)\right\|_{L^{1}\left(\mathbb{R}^{5}\right)}$ as

$$
\frac{a_{2}}{2}\left\|v_{1}-v_{2}\right\|_{L^{2}\left(\mathbb{R}^{5}\right)}\left\|2 u_{0}+v_{1}+v_{2}\right\|_{L^{2}\left(\mathbb{R}^{5}\right)} \leq a_{2}\left\|v_{1}-v_{2}\right\|_{H^{3}\left(\mathbb{R}^{5}\right)}\left(\left\|u_{0}\right\|_{H^{3}\left(\mathbb{R}^{5}\right)}+1\right) .
$$

Hence we obtain the upper bound for the norm $\left\|u_{1}(x)-u_{2}(x)\right\|_{L^{2}\left(\mathbb{R}^{5}\right)}^{2}$ as

$$
\varepsilon^{2}\|\mathcal{K}\|_{L^{1}\left(\mathbb{R}^{5}\right)}^{2}\left\|v_{1}-v_{2}\right\|_{H^{3}\left(\mathbb{R}^{5}\right)}^{2}\left\{\frac{a_{2}^{2}}{(2 \pi)^{5}}\left(\left\|u_{0}\right\|_{H^{3}\left(\mathbb{R}^{5}\right)}+1\right)^{2}\left|S_{5}\right| R+\frac{a_{1}^{2}}{R^{4}}\right\} .
$$

Let us use Lemma 4 to minimize the expression above over $R>0$ to derive that $\left\|u_{1}(x)-u_{2}(x)\right\|_{L^{2}\left(\mathbb{R}^{5}\right)}^{2}$ is bounded above by

$$
\varepsilon^{2}\|\mathcal{K}\|_{L^{1}\left(\mathbb{R}^{5}\right)}^{2}\left\|v_{1}-v_{2}\right\|_{H^{3}\left(\mathbb{R}^{5}\right)}^{2} \frac{5}{4^{\frac{4}{5}}} \frac{a_{2}^{\frac{8}{5}}}{(2 \pi)^{4}}\left(\left\|u_{0}\right\|_{H^{3}\left(\mathbb{R}^{5}\right)}+1\right)^{2}\left|S_{5}\right|^{\frac{4}{5}} a_{1}^{\frac{2}{5}} .
$$

(2.7) and (2.8) yield

$$
\nabla(-\Delta)\left(u_{1}-u_{2}\right)=\varepsilon \nabla \int_{\mathbb{R}^{5}} \mathcal{K}(x-y)\left[g\left(u_{0}(y)+v_{1}(y)\right)-g\left(u_{0}(y)+v_{2}(y)\right)\right] d y,
$$


such that

$$
\left\|\nabla(-\Delta)\left(u_{1}-u_{2}\right)\right\|_{L^{2}\left(\mathbb{R}^{5}\right)}^{2} \leq \varepsilon^{2}\|\mathcal{K}\|_{L^{1}\left(\mathbb{R}^{5}\right)}^{2}\left\|\nabla g\left(u_{0}+v_{1}\right)-\nabla g\left(u_{0}+v_{2}\right)\right\|_{L^{2}\left(\mathbb{R}^{5}\right)}^{2} .
$$

Let us express $\nabla g\left(u_{0}+v_{1}\right)-\nabla g\left(u_{0}+v_{2}\right)$ as

$$
\begin{gathered}
g^{\prime}\left(u_{0}+v_{1}\right)\left(\nabla u_{0}+\nabla v_{1}\right)-g^{\prime}\left(u_{0}+v_{2}\right)\left(\nabla u_{0}+\nabla v_{2}\right)= \\
=\left(\nabla u_{0}+\nabla v_{1}\right) \int_{u_{0}+v_{2}}^{u_{0}+v_{1}} g^{\prime \prime}(s) d s+\left(\nabla v_{1}-\nabla v_{2}\right) \int_{0}^{u_{0}+v_{2}} g^{\prime \prime}(s) d s .
\end{gathered}
$$

This gives us the upper bound for $\left|\nabla g\left(u_{0}+v_{1}\right)-\nabla g\left(u_{0}+v_{2}\right)\right|$ as

$$
\sup _{s \in I}\left|g^{\prime \prime}(s)\right|\left|v_{1}-v_{2}\right|\left|\nabla u_{0}+\nabla v_{1}\right|+\sup _{s \in I}\left|g^{\prime \prime}(s)\right|\left|u_{0}+v_{2}\right|\left|\nabla v_{1}-\nabla v_{2}\right| .
$$

This expression can be easily estimated from above via the Sobolev embedding (1.5) by

$$
a_{2} c_{e}\left\|v_{1}-v_{2}\right\|_{H^{3}\left(\mathbb{R}^{5}\right)}\left|\nabla u_{0}+\nabla v_{1}\right|+a_{2} c_{e}|| u_{0}+v_{2} \|_{H^{3}\left(\mathbb{R}^{5}\right)}\left|\nabla v_{1}-\nabla v_{2}\right| .
$$

Thus, by means of (2.5) using that $v_{1} \in B_{\rho}$ we arrive at the upper bound for $\left\|\nabla(-\Delta)\left(u_{1}-u_{2}\right)\right\|_{L^{2}\left(\mathbb{R}^{5}\right)}^{2}$ as

$$
4 \varepsilon^{2}\|\mathcal{K}\|_{L^{1}\left(\mathbb{R}^{5}\right)}^{2} a_{2}^{2} c_{e}^{2}\left(\left\|u_{0}\right\|_{H^{3}\left(\mathbb{R}^{5}\right)}+1\right)^{2}\left\|v_{1}-v_{2}\right\|_{H^{3}\left(\mathbb{R}^{5}\right)}^{2} .
$$

Estimates (2.9) and (2.10) yield that the norm $\left\|u_{1}-u_{2}\right\|_{H^{3}\left(\mathbb{R}^{5}\right)}$ is bounded from above by

$$
\varepsilon\|\mathcal{K}\|_{L^{1}\left(\mathbb{R}^{5}\right)}\left(\left\|u_{0}\right\|_{H^{3}\left(\mathbb{R}^{5}\right)}+1\right) a_{2}^{\frac{4}{5}}\left[\frac{5}{4^{\frac{4}{5}}} \frac{a_{1}^{\frac{2}{5}}}{(2 \pi)^{4}}\left|S_{5}\right|^{\frac{4}{5}}+4 a_{2}^{\frac{2}{5}} c_{e}^{2}\right]^{\frac{1}{2}}\left\|v_{1}-v_{2}\right\|_{H^{3}\left(\mathbb{R}^{5}\right)} .
$$

Hence, the map $T_{g}: B_{\rho} \rightarrow B_{\rho}$ defined by problem (1.12) is a strict contraction for all values of $\varepsilon>0$ small enough. Its unique fixed point $u_{p}(x)$ is the only solution of problem (1.10) in $B_{\rho}$ and the resulting $u(x) \in H^{3}\left(\mathbb{R}^{5}\right)$ given by (1.9) is a solution of equation (1.2).

Acknowledgements. Stimulating discussions with D.Pelinovsky are gratefully acknowledged.

\section{References}

[1] G.L. Alfimov, E.V. Medvedeva, D.E. Pelinovsky, Wave Systems with an Infinite Number of Localized Traveling Waves, Phys. Rev. Lett., 112 (2014), 054103, 5pp. 
[2] C. Amrouche, V. Girault, J. Giroire, Dirichlet and Neumann exterior problems for the n-dimensional Laplace operator. An approach in weighted Sobolev spaces, J. Math, Pures Appl., 76 (1997), 55-81.

[3] C. Amrouche, F. Bonzom, Mixed exterior Laplace's problem, J. Math. Anal. Appl., 338 (2008), 124-140.

[4] P. Bolley, T.L. Pham, Propriété d'indice en théorie Holderienne pour des opérateurs différentiels elliptiques dans $R^{n}$, J. Math. Pures Appl., 72 (1993), $105-119$.

[5] P. Bolley, T.L. Pham, Propriété d'indice en théorie Hölderienne pour le problème extérieur de Dirichlet, Comm. Partial Differential Equations, 26 (2001), No. 1-2, 315-334.

[6] N. Benkirane, Propriété d'indice en théorie Holderienne pour des opérateurs elliptiques dans $R^{n}$, CRAS, 307, Série I (1988), 577-580.

[7] S. Cuccagna, D. Pelinovsky, V. Vougalter, Spectra of positive and negative energies in the linearized NLS problem, Comm. Pure Appl. Math., 58 (2005), No. 1, 1-29.

[8] H.L. Cycon, R.G. Froese, W. Kirsch, B. Simon, Schrödinger Operators with Application to Quantum Mechanics and Global Geometry, Springer-Verlag, Berlin (1987).

[9] A. Ducrot, M. Marion, V. Volpert, Systemes de réaction-diffusion sans propriété de Fredholm, CRAS, 340 (2005), 659-664.

[10] A. Ducrot, M. Marion, V. Volpert, Reaction-diffusion problems with non Fredholm operators, Advances Diff. Equations, 13 (2008), No. 11-12, 11511192.

[11] E. Lieb, M. Loss, Analysis. Graduate Studies in Mathematics, 14, American Mathematical Society, Providence (1997).

[12] V. Volpert. Elliptic partial differential equations. Volume 1. Fredholm theory of elliptic problems in unbounded domains. Birkhauser, 2011.

[13] V. Vougalter, On threshold eigenvalues and resonances for the linearized NLS equation, Math. Model. Nat. Phenom., 5 (2010), No. 4, 448-469.

[14] V. Volpert, B. Kazmierczak, M. Massot, Z.Peradzynski, Solvability conditions for elliptic problems with non-Fredholm operators, Appl.Math., 29 (2002), No. 2, 219-238. 
[15] V. Vougalter, V. Volpert, Solvability conditions for some non-Fredholm operators, Proc. Edinb. Math. Soc. (2), 54 (2011), No.1, 249-271

[16] V. Vougalter, V. Volpert. On the solvability conditions for some non Fredholm operators, Int. J. Pure Appl. Math., 60 (2010), No. 2, 169-191.

[17] V. Vougalter, V. Volpert. On the solvability conditions for the diffusion equation with convection terms, Commun. Pure Appl. Anal., 11 (2012), No. 1, 365-373.

[18] V. Vougalter, V. Volpert. Solvability relations for some non Fredholm operators, Int. Electron. J. Pure Appl. Math., 2 (2010), No. 1, 75-83.

[19] V. Volpert, V. Vougalter. On the solvability conditions for a linearized CahnHilliard equation, Rend. Istit. Mat. Univ. Trieste, 43 (2011), 1-9.

[20] V. Vougalter, V. Volpert. On the existence of stationary solutions for some nonFredholm integro-differential equations, Doc. Math., 16 (2011), 561-580.

[21] V. Vougalter, V. Volpert. Solvability conditions for a linearized Cahn-Hilliard equation of sixth order, Math. Model. Nat. Phenom., 7 (2012), No. 2, 146-154.

[22] V. Vougalter, V. Volpert. Solvability conditions for some linear and nonlinear non-Fredholm elliptic problems, Anal. Math. Phys., 2 (2012), No.4, 473-496.

\section{Appendix}

We used in this work solvability conditions for linear elliptic equations in $\mathbb{R}^{d}$ obtained in [22]. For the convenience of the readers we formulate them here. We study the existence of solutions of the linear equation

$$
-\Delta \phi-\omega \phi=-h(x), \omega \geq 0
$$

with a square integrable right side in the space $H^{2}\left(\mathbb{R}^{d}\right), d \in \mathbb{N}$ equipped with the norm

$$
\|u\|_{H^{2}\left(\mathbb{R}^{d}\right)}^{2}:=\|u\|_{L^{2}\left(\mathbb{R}^{d}\right)}^{2}+\|\Delta u\|_{L^{2}\left(\mathbb{R}^{d}\right)}^{2} .
$$

Lemma 5. Let $h(x) \in L^{2}(\mathbb{R})$. The the following assertions hold:

a) When $\omega>0$ and $x h(x) \in L^{1}(\mathbb{R})$ problem (3.1) admits a unique solution in $H^{2}(\mathbb{R})$ if and only if

$$
\left(h(x), \frac{e^{ \pm i \sqrt{\omega} x}}{\sqrt{2 \pi}}\right)_{L^{2}(\mathbb{R})}=0 .
$$


b) When $\omega=0$ and $x^{2} h(x) \in L^{1}(\mathbb{R})$ problem (3.1) admits a unique solution in $H^{2}(\mathbb{R})$ if and only if

$$
(h(x), 1)_{L^{2}(\mathbb{R})}=0,(h(x), x)_{L^{2}(\mathbb{R})}=0 .
$$

Lemma 6. Let $h(x) \in L^{2}\left(\mathbb{R}^{d}\right), d \geq 2$. The the following assertions hold:

a) When $\omega>0$ and $x h(x) \in L^{1}\left(\mathbb{R}^{d}\right)$ problem (3.1) admits a unique solution in $H^{2}\left(\mathbb{R}^{d}\right)$ if and only if

$$
\left(h(x), \frac{e^{i p x}}{(2 \pi)^{\frac{d}{2}}}\right)_{L^{2}\left(\mathbb{R}^{d}\right)}=0, p \in S_{\sqrt{\omega}}^{d} \text { a.e., } d \geq 2 .
$$

b) When $\omega=0$ and $|x|^{2} h(x) \in L^{1}\left(\mathbb{R}^{2}\right)$ problem (3.1) admits a unique solution in $H^{2}\left(\mathbb{R}^{2}\right)$ if and only if

$$
(h(x), 1)_{L^{2}\left(\mathbb{R}^{2}\right)}=0,\left(h(x), x_{k}\right)_{L^{2}\left(\mathbb{R}^{2}\right)}=0,1 \leq k \leq 2 .
$$

c) When $\omega=0$ and $|x| h(x) \in L^{1}\left(\mathbb{R}^{d}\right), d=3,4$ problem (3.1) admits a unique solution in $H^{2}\left(\mathbb{R}^{d}\right)$ if and only if

$$
(h(x), 1)_{L^{2}\left(\mathbb{R}^{d}\right)}=0, d=3,4 .
$$

d) When $\omega=0$ and $|x| h(x) \in L^{1}\left(\mathbb{R}^{d}\right), d \geq 5$ problem (3.1) possesses a unique solution in $H^{2}\left(\mathbb{R}^{d}\right)$.

Lemma 7. Let $\omega=0$ and $h(x) \in L^{1}\left(\mathbb{R}^{d}\right) \cap L^{2}\left(\mathbb{R}^{d}\right)$ with $d \geq 5$. Then problem (3.1) admits a unique solution in $H^{2}\left(\mathbb{R}^{d}\right)$. 